\title{
DE CÓMO OCCIDENTE DILUYÓ LOS CONOCIMIENTOS EN SALUD DE LAS MUJERES. REPERCUSIONES EN EL CASO DE MÉXICO
}

\author{
Georgina Sánchez-Ramírez \\ El Colegio de la Frontera Sur - México \\ gsanchez@ecosur.mx
}

Recibido: 24-02-2010

Aceptado: 16-04-2010

\section{Resumen}

La mayoría de las mujeres en los países subdesarrollados procuran su salud sin autonomía. El objeto de este trabajo es hacer un análisis a través de la historia de la dilución de los saberes de las mujeres respecto a la salud y el conocimiento de su propio cuerpo, como una herencia de la Práctica Médica occidental. La información se obtuvo de bibliografía especializada en el tema, elaborándose con ella un análisis a partir del enfoque de género y salud, con el cual se muestra cómo desde la belladona hasta la partera, los conocimientos de las mujeres se siguen considerando marginales en el marco de la medicina hegemónica.

Palabras claves: Práctica Médica, mujeres y salud, género y salud, mujeres en México.

\begin{abstract}
Most women in underdeveloped countries search for their health without autonomy. The object of this work is to analyze through history the dilution of women's knowledge about health and knowledge of their own body, as a legacy from the Western Medical Practice. The information is based on specialized literature, thus elaborating an analysis by the focus on gender and health, which shows how from the belladonna to
\end{abstract}


the midwife, women's knowledge is still considered marginal in the framework of the hegemonic medicine.

Key words: Medical practice, women and health, gender and health, women in Mexico.

\section{Introducción}

La preocupación por el equilibrio entre salud y enfermedad son producto del devenir histórico de las diferentes regiones y culturas, y la procuración de la misma ha dependido de los recursos de los que se disponen tanto a nivel general como particular.

En la época contemporánea se intenta trabajar de manera más universal desde el enfoque consensuado a través de la Organización Mundial de la Salud (OMS), salud "no solo como la ausencia de enfermedad, sino el estado de bienestar físico, mental y social", además de lo mencionado en la Carta de Ottawa (signada por 30 países en el año de 1986) donde se establece que "para alcanzar un estado adecuado de bienestar físico, mental y social un individuo o grupo debe de ser capaz de identificar y realizar sus aspiraciones, satisfacer sus necesidades y modificar o adaptarse al medio ambiente" (OMS, 1987:77) y con elementos que, desde el enfoque de género y salud, han intentado recoger lo que los movimientos de mujeres proclaman desde los años 80's en torno a la calidad de salud de las mujeres, definida como aquella que además de resolver el motivo de la consulta, contribuye a mejorar positivamente la baja autoestima, así como a promover la apropiación de la corporalidad y el ejercicio de sus derechos (Matamala,1995).

No obstante es una meta que dista de lograrse, ocupando un papel determinante la historia que subyace a la consolidación de la medicina hegemónica como tal, y sus correspondientes implicaciones en la casi desaparición o devaluación de los conocimientos que las mujeres habían manejado sobre su cuerpo y otras prácticas de sanación diferentes a las reconocidas por occidente. Prácticas que, hasta hoy en día, 
resultan en sincretismos de conocimientos en la búsqueda del equilibrio entre salud y enfermedad, y que prevalecen con mayor intensidad en las regiones no desarrolladas, como es el caso de la mayor parte de México.

En este trabajo se presenta una breve reseña histórica, resultado de la revisión de documentos respecto a la desvalorización de los saberes femeninos sobre el cuidado de la salud; hecho que en México supuso la incorporación de la medicina occidental con su racionalidad técnica y su sincretismo con la medicina tradicional. Desafortunadamente para México no hay documentos que precisen cómo ha sido este proceso tal y como se encuentran investigaciones para Europa, sin embargo si se pueden realizar análisis de los efectos que todo ello ha provocado en la atención sanitaria de las mujeres mexicanas en la época contemporánea.

\section{Algunos aspectos sobre la legitimación de la medicina académica}

Desde siempre y al parecer en todas las culturas, han coexistido la salud y la enfermedad. La correspondiente diversidad de alternativas y respuestas para lograr alejarse del daño y acercarse a lo vital, hace casi imposible hablar de una respuesta única ante los procesos de enfermedad, encontrándose modelos imperantes sobre otros ya existentes, pero nunca esquemas únicos. El caso de México, es un ejemplo de la confluencia de diversidades, producto de la transculturación provocada por la conquista europea y la constante influencia tanto de Oriente como de Occidente., sin embargo se consideraron desde entonces y hasta la fecha, prácticas alternativas, sin un reconocimiento legítimo como métodos de curación.

Tal y como lo mencionan Sayavedra y Flores (1997: 69), en contextos como el de México es más acertado hablar de una Práctica Médica o hegemónica dominante, interpretando a la Práctica Médica, como "el conjunto de conocimientos y prácticas sociales con las que una sociedad determinada históricamente, intenta dar respuesta a 
los procesos de salud y enfermedad", la cual persiste y se mantiene a través de los grupos hegemónicos que la protegen, lo que provoca a su vez:

- La formación de conocimiento que sedimentándose al ser transmitido por diferentes niveles (del básico al profesional), llega a considerarse "sentido común”, acreditado y garantizado por la sociedad.

- El control (a través de esos conocimientos legitimados) de las instituciones de salud públicas y privadas, seleccionando las tecnologías que requieren para otorgar sus servicios, con recursos económicos que lo sustentan en cada una de sus áreas y niveles (Sayavedra, 1982).

Debido a la manera en que la Práctica Médica está estructurada, produce un acceso diferencial no equitativo de los grupos sociales, con discriminación por raza, etnia, edad, y sexo; lo cual es una triste realidad en México, en donde la salud pública está transitando hacia un esquema de salud privada, cobrándose una cuota por la atención recibida aún en hospitales públicos bajo un programa del gobierno mexicano denominado Seguro Popular, que es un seguro de gastos médicos para las clases más pobres, pero que además no distingue entre las necesidades diferenciales de las y los usuarios por sexo, edad, etnia, u otras particularidades, ni tampoco considera prácticas médicas de curación diferentes a las hegemónicas occidentales.

Pero ¿cuáles son los antecedentes de dicha Práctica Médica? Sayavedra y Flores (1997) hacen una síntesis remontándose a la Grecia clásica en donde existieron básicamente dos tendencias contradictorias en la procuración de la salud: una atribuida a la diosa Higia y la otra a Asclepcio o Esculapio, Hijo de Apolo.

La tendencia atribuida a Higia define la salud como "un atributo positivo al cual las personas tienen derecho si gobiernan sus vidas sabiamente" (Sayavedra y Flores, 1997:70), por lo que una de las funciones de la medicina era descubrir y enseñar las leyes naturales que aseguraban a las personas un bienestar de cuerpo y mente, sin limitarse a medidas preventivas ya que se consideraba de suma importancia tomar en cuenta aspectos humanos e históricos de la cultura a la cual se pertenece. 
Así mismo, Sayavedra y Flores (1997) mencionan que, la tendencia atribuida a Asclepcio fue personificada por Hipócrates, y en ella el papel principal del "cuidador o procurador" de la salud recae en el profesional o médico, en donde prevalece la “corrección” de las imperfecciones invocándose a la Diosa Panacea (conocedora de medicamentos naturales).

Como producto de esta tendencia hipocrática surge o se concreta un gran interés por el diagnóstico y la curación, estableciéndose así una serie de técnicas, formas y procedimientos que continúan vigentes hasta ahora en la medicina occidental, donde la curación se prioriza sobre la prevención, y por lo tanto la medicación de las personas, antes de considerar seriamente los cambios en el entorno social y su posible influencia en las mismas.

Dentro de este devenir histórico de más de 20 siglos, tuvo un papel determinante en el fanatismo cristiano europeo, por lo menos hasta bien entrado el siglo XVII, la interpretación dada al médico Galeno y la explicación de la causa de las enfermedades por medio de su teoría de los humores corporales, que tenía como trasfondo la filosofía natural aristotélica, con la cual se atribuía los padecimientos a cuestiones como "la corrupción de los humores, la alteración de las condiciones anatómicas, la presencia de materia pecaminosa" (Bernabeu, Barona, Perdiguero y Robles, 1999: 105).

Esto tuvo consecuencias no del todo venturosas, desde la Edad Media, en la que el fanatismo religioso católico inculcó medidas poco higiénicas sobre el cuidado del cuerpo hasta la Inquisición, en donde se torturó y dio muerte a muchos de los y las que intentaron investigar o intervenir en salud desde otras perspectivas no galénicas ${ }^{1}$.

Ciencia e Iglesia habían estado íntimamente ligadas hasta entonces, lo cual cambió radicalmente al modificarse las estructuras de poder y consolidarse los preceptos científicos de Galileo y Copérnico en los siglos XVII y XVIII a través de diversos pensadores, destacando las leyes universales de Newton y el discurso de la

\footnotetext{
${ }^{1}$ Véase al respecto Guzmán, 1987; el hace una lectura crítica sobre el cuerpo y su cuidados dese el siglo VI hasta el XIX de la era cristiana, en América.
} 
razón de Descartes. A este último se le atribuye la separación de mente-materia, imponiéndose con ello la razón sobre el resto de las formas de conocimiento (Esteban, 2001).

En el caso de Mesoamérica y Centroamérica como una de las secuelas de la colonización, se legitimaron a lo largo del tiempo, frente a las Prácticas Médicas existentes en el "Nuevo Mundo", el conjunto de prácticas, conocimientos y teorías occidentales; primero por la coerción y después por la "razón", en pueblos que tenían una visión muy diferente de la salud (entendida más desde la tendencia de Higia).

Fue entonces como se instauró y legitimó poco a poco la Práctica Médica occidental como única o universal, controlada además por el Estado, aunque con algunas recuperaciones del conocimiento médico mesoamericano preexistente gracias al trabajo de amanuenses indígenas, criollos y españoles ${ }^{3}$, y como parte de necesidades reales de atención para quienes no tenían recursos (la población india).

Se calcula que aproximadamente solo un $5 \%$ de la población vivía en las ciudades, por tanto el alcance de los médicos occidentales, que no solo eran extremadamente escasos, y no salían de las ciudades, fue muy limitado. A cambio los monjes y monjas con sus conocimientos, sus jardines de hierbas curativas, no estaban tan lejos de las prácticas prehispánicas, y quienes definitivamente no tenían acceso ni a médicos occidentales ni a monjes o monjas, acudieron con curanderos y curanderas indígenas.

Sayavedra y Flores (1997) mencionan también como en Occidente, el Renacimiento (hacia el Siglo XV) permitió el avance de la práctica médica en cuanto a las causas de las enfermedades, contribuyendo al desarrollo posterior de las teorías microbianas o contagionistas del siglo XIX. No hay que olvidar que las cifras de

\footnotetext{
${ }^{2}$ Véase al respecto: Aguirre y Lozoya et al., 1989.

${ }^{3}$ Un ejemplo de ello es el "Libelus Medicinalis", documento escrito en latín por un indígena letrado en la época colonial de México aproximadamente hacia el año 1522, que trata sobre el conocimiento de etnobotánica acumulado hasta ese entonces. El manuscrito fue devuelto a México 300 años después por el Papa y en 1964, M. De la Cruz lo reeditó en castellano auspiciado por el Instituto Mexicano del Seguro Social.
} 
mortalidad y morbilidad tuvieron una disminución sin precedentes, ante el hallazgo de microbios y vacunas.

Pardo (1998) indica en ese mismo sentido, que la consolidación de la medicina científica occidental entre los pasados siglos XIX y XX, se debió también al descubrimiento de antibióticos y la invención de la anestesia, la cual impulsó grandemente las intervenciones quirúrgicas, así como el tratamiento de los traumatismos y las infecciones. Dicho proceso se aceleró más a partir de las dos guerras mundiales; desde entonces, todo lo que no se refiriera a "los profesionales" en biomedicina fue etiquetado peyorativamente como curanderismo.

Para el caso de la salud de las mujeres en particular, un avance de suma importancia para disminuir la mortalidad femenina postparto según Sayavedra y Flores (1997) fue el encontrar la causa de la "fiebre puerperal", tan común por la falta de higiene de los médicos en el momento del parto.

A pesar de todo, esa euforia de avances tuvo a su vez un costo social como contrapartida, ya que se abandonaron otras investigaciones en salud más integrales como la homeopática o del anticontagio de Virchow; ésta última hacía más énfasis en la sociedad y no sólo en el individuo, postulando reformas sociales como asuntos de salud.

Sin embargo, y como se mencionaba al principio de este trabajo, la Práctica Médica hegemónica no se ha mantenido rígida y lineal, ya que ha incorporado en el tiempo además de importantes avances científicos y técnicos, otras teorías, incluso prácticas curativas subalternas, mismas que son definidas por Sayavedra y Flores (1997: 75) como "todas aquellas [prácticas] que desde la exclusión mantienen una propuesta conformada por una serie de elementos teóricos y prácticos que puedan estar integrados o no en un saber reconocido como tal".

Estas prácticas se han mantenido desorganizadas ya que si bien en un principio eran manejadas solamente por personas expertas. La dificultad de generar, mantener y controlar los conocimientos de manera teórica y sistematizada ha permitido un intrusismo en dichas prácticas, dando como resultado una práctica empirista poco responsable. 
En México destacan los conocimientos preexistentes a la colonización, que son los más difundidos en el país hoy en día, y que continúan vigentes en zonas rurales y urbanas por parte de curanderos y parteras profesionales, recreadores del saber acumulado por siglos; de hecho casi en todos los mercados de alimentos, tanto fijos como ambulantes, hay una zona para el comercio de productos herbolarios destinados a la curación de distintos padecimientos.

En relación con la práctica creciente de empiristas, existe un saber enajenado atribuido a "yerberos" o a mujeres que curan a nivel doméstico, principalmente a sus familiares o a personas de su entorno más cercano. Este fenómeno lejos de desaparecer con el tiempo está tendiendo a incrementarse como resultado de la recesión económica en la que vive México, lo cual constriñe la disposición de recursos económicos para el cuidado de la salud; y las personas tanto en zonas rurales como urbanas acuden a curanderos o curanderas para resolver los males que les aquejan; sin embargo no todas las personas que practican este tipo de medicina están calificadas para ello; como resultado de la subalternidad en la que han permanecido este tipo de prácticas.

De este modo, puede apreciarse que la Práctica Médica imperante en Mesoamérica y Centroamérica fue usurpada por la Práctica Médica hegemónica de Occidente aunque no desaparecieron las prácticas tradicionales del "Nuevo Mundo". Al brotar entre la población indígena padecimientos antes desconocidos traídos de Occidente y viceversa, surgió una amalgama de prácticas terapéuticas.

Menéndez (1983) menciona que eso transformó el trabajo de los llamados "curanderos o curanderas", complejizándose la visión de la causalidad de las enfermedades, y persistiendo hasta hoy en día una especie de contraposición entre las causas mágico-místicas de las prácticas médicas subalternas mesoamericanas y las causas fundamentadas en lo racional-naturalista de la Práctica Médica de Occidente.

No obstante, en ninguna de las dos prácticas médicas se consideró una diferencia entre las condiciones de varones y mujeres frente a las enfermedades aunque si hacen una distinción por sexo. Sayavedra y Flores (1997) y Blazquez (2008) mencionan que ese proceso no solo no visibilizó las diferentes condiciones de la 
población de cara a los padecimientos sino que además, se ilegitimó a las mujeres como sanadoras profesionales, a pesar de que las mujeres rechazan figuras como el ejercicio de la partería por varones.

Persiste aún un poder médico masculino, cuya base es la certeza de que los varones están más ligados a la razón, a la objetividad, al mundo público, mientras la contraparte la conforman las mujeres con sus pensamientos subjetivos, su "cuerpo perecedero", enfrascadas en el mundo privado o mejor dicho, mundo doméstico. Desafortunadamente esta desigualdad se aprecia incluso en quienes realizan prácticas subalternas de sanación en México; en donde a pesar de ser más las mujeres reconocidas como sanadoras; son los curanderos los que suelen gozar de mayor prestigio y de un cobro mayor por sus servicios ${ }^{4}$.

Esta diferenciación entre "los mundos" en que se desenvuelven varones y mujeres tiene como origen la división sexual del trabajo y la correspondiente separación de esferas: la pública en donde tiene lugar el prestigio y reconocimiento de los actos que en ella se realizan, y la privada, en donde el trabajo que se realiza es ignorado por la sociedad, lo cual en contextos como México tiene un peso mayor; ya que los roles tradicionales de género segregan a las mujeres a espacios privados considerados femeninos y que si bien varían de acuerdo a la condición de vida de las mujeres, afectan su posición como género en todo el país.

Murillo (1996) apunta hacia una precisión de términos aclarando que privado no es igual que doméstico, ya que el término "privado" adopta por lo menos dos significados diferentes entre sí, definidos por la autora como: "apropiación de sí mismo" como beneficio, producto de un tiempo (mayoritariamente aprovechado por los varones) y como "privación de sí" que significaría estar al servicio continuo de las demandas de los otros, lo cual se aplica a la vida o ámbito de lo doméstico, y negarse a ello sería calificado por el entorno como un "acto imperdonable de egoísmo".

\footnotetext{
${ }^{4}$ Véase al respecto Marcos (1989), quien hace una descripción muy cercana al trabajo de las sanadoras en una zona de México.
} 
Así vemos la importancia de "medir" el tiempo que las mujeres dedican al cuidado de los demás, como un indicador de salud, indispensable para entender el desgaste físico y emocional de la población femenina en edades reproductivas y postreproductivas.

Murillo menciona además que se ha hecho una asociación unidireccional entre privado-doméstico-femenino, como si la privacidad femenina no tuviera cabida "la autonomía e individuación [de las mujeres] han estado subordinas al papel de ayudante, nutriente y compañera" (1996: 20), por tanto la privacidad se convierte en un lujo para las mujeres, trascendiendo culturas.

Desafortunadamente las pautas culturales de socialización vigentes hasta ahora en México, continúan adiestrando a las mujeres en el "cuidado de los otros", sin una acertada valoración del tiempo dedicado a los demás y a años luz del "autocuidado", como símbolo de la anhelada pertenencia de sí misma.

\section{La medicina y su racionalidad técnica: la deslegitimación de los saberes femeninos}

El origen y causas de la exclusión de las mujeres del mundo público y la correspondiente deslegitimación de sus saberes, es hasta cierto punto una incógnita. Sendón (1988:15) hace un vertiginoso recorrido histórico desde ocho mil años antes de la era Cristiana, en un intento por descifrar el misterio de la pérdida del reconocimiento y valoración de los conocimientos femeninos siendo excluidos del mundo público y del poder que representan: "Los primeros mitos que conocemos son de carácter femenino y corresponden a la saga de las diosas madres "pero después de milenios... serán los mitos de carácter masculino los que impondrán su peculiar orden simbólico...”.

Florinda Riquer (1989), realiza un vertiginosos recorrido que arroja algunas luces respecto al por qué las deslegitimaciones de los saberes de las mujeres, situándose en la Europa del siglo XIV, en donde la caza de personas acusadas de brujería cobra auge como nunca antes; si bien pocos historiadores hacen una distinción entre el 
número de acusadas versus acusados por brujería, por ejemplo Harris menciona que "500 000 personas fueron declaradas culpables de brujería y murieron quemadas en Europa entre los siglos XV y XVII [...]" (1981: 181). No obstante no aclara la distinción por sexo de las víctimas lo cual es un hecho relevante dado el contexto en el que se situaron estos hechos.

Se trataba de un momento histórico en los que peligraba el poder monolítico del catolicismo romano, y donde toda sospecha de oposición al status quo fue etiquetada como herejía, cuyo castigo (previa confesión bajo tortura) fue la muerte, una vez que se había delatado a otras personas como practicantes de hechizos o embrujos, y en donde no se trataba solamente de aquellas personas que realizaban prácticas curativas; sino de todo aquel ser que osaba transgredir el orden social y religioso establecido.

Aunado a esto las mujeres más que los hombres fueron excluidas de las instituciones oficiales del conocimiento (universidades) relegándolas exclusivamente a la práctica empírica de sus conocimientos en espacios ligados a los ciclos naturales donde "seguían escuchando las voces de otros habitantes de bosques y praderas que ayudaban a bien morir, a amar, a colaborar en el nacimiento o a curar gracias a las propiedades de las flores" (Riquer, 1989: 339). Era la adecuación de las propias mujeres a una realidad simbólica que no podían transformar de manera directa; sino solamente a través de la preservación de prácticas heredadas de sus ancestras, lo cual sigue ocurriendo con mujeres sanadoras en México.

Sendón indica que durante esa etapa de inconformidad social respecto al orden establecido por parte de la iglesia católica, se consolidó el fenómeno del "satanismo", mezcla de imaginación popular y de los deseos sublimados del clero cristiano y demás profesionistas poderosos. "En aquella locura de caza de brujas no solo los teólogos o los inquisidores se mostraban prolijos para adivinar la mínima señal que delatara a la víctima, sino que una nube de médicos, jueces, abogados y comerciantes se sumó al rastreo" (1988: 123). ¿No eran éstos indicios de que lo que se pretendía era hacer desaparecer todo aquello que pusiera en duda el orden establecido, incluido el poder de los varones sobre las mujeres? 
Pero ¿cuál es la relación entre brujería y sanación? Tanto Michelet (1987) como Riquer (1989) hacen alusión a que en Europa entre los siglos XIII y XV, la lepra, la epilepsia y la sífilis se abrieron un macabro paso a través del hambre y la anemia persistente en la población, que no tenía acceso a los exclusivos médicos árabes o judíos de las cortes occidentales.

La resignación ante dolorosas muertes, remedio ofrecido por los sacerdotes católicos, no convencía a la muchedumbre con ansias humanas de sobrevivir. Sobre la lepra, el citado autor menciona:

"La guerra que la Edad Media declaró a la carne y a la limpieza tenía que dar frutos. Más de una santa es ponderada por no haberse lavado las manos nunca. ¡Y cuánto menos el resto del cuerpo! La desnudez momentánea hubiera sido un pecado mortal ¡Ningún baño durante mil años! [...] De aquí procede un cruel accidente tan poco poético en plena novela, los furiosos picores del siglo XIII [...]" (Michelet, 1987:121).

El recurrir a las belladonas (o "buenas mujeres" como se les solía llamar a las "brujas" por temor) que mediante pócimas y remedios podían por lo menos mitigar los dolores, comenzó a ser una práctica cada vez más difundida entre el pueblo.

Michelet a su vez menciona que Paracelso (uno de los médicos más reconocidos del Renacimiento) declaró que cuanto sabía de medicina popular, le había sido trasmitido por las buenas mujeres "cuando éste quemó toda la medicina en Basilea, en 1527, afirmó no saber más que lo que le habían enseñado las brujas [...]” (1987: 3233).

En el libro que Paracelso escribió sobre "Las Enfermedades de las Mujeres" (el primero del que se tiene conocimiento en Occidente sobre el tema) es evidente, según Michelet (1987: 123) la experiencia trasmitida por las "brujas", que eran a quienes acudían las mujeres para sanar "En ésta época, la mujer nunca admitió un médico 
masculino, nunca se confió a él [...] Las brujas eran las únicas observadoras en este campo y fueron para la mujer, la sola y única medicina $[\ldots]$ ”.

Desafortunadamente y como en todo proceso, llegó la decadencia. Ésta se debió a varios factores; por un lado estaba la persecución del clero sobre las belladonas, que a todas luces se perfiló como "[...] una cuestión de poder, ya que las brujas ejercían un gran ascendente sobre la gente del pueblo por quienes eran consultadas, tanto en los problemas del espíritu, como en los de cuerpo [...]” (Sendón, 1998:117).

Coincidiendo con Riquer (1989), no se puede ser contundente respecto a la intención de exterminar a las mujeres por el hecho de poseer conocimientos, ya que desafortunadamente, las víctimas no fueron exclusivamente mujeres; pero los sacerdotes no despreciaron el ocupar el lugar que estas mujeres tenían de consejeras y sanadoras; al quemarlas y poner al pueblo en su contra, los sacerdotes se convirtieron en los “curas", los sanadores de cuerpos y almas, a través de un poder legitimado por el terror y el exterminio, con una marcada tendencia de rechazo hacia la sexualidad como dimensión humana y hacia el reconocimiento de las mujeres como seres capaces de saber, placer y poder.

Por el otro lado, está lo que menciona Michelet (1987) respecto a que comenzaron a surgir abundantes acusaciones de brujería sobre todo aquello que se "envidiaba" o se percibía como amenaza a lo establecido. El saber se enajena y prolifera la superchería, como una manera de burla hacia la sociedad que no defiende a sus sanadoras, además el comercio de filtros y brebajes reditúa.

Otro más de los desprestigios sobre la imagen de las brujas según Michelet fue la consecuencia de ser "protegidas" por algún señor o amo, por quien, bajo amenaza de denunciarlas ante la Iglesia y sus terribles tribunales inquisidores, se veían obligadas a cumplir sus caprichos (robar, secuestrar, matar, etc.), sin ser "diabólicamente" poderosas como para escapar de ese infierno terrenal.

Tanto Greer (1993) como Sayavedra y Flores (1997) y Blázquez (2008) mencionan que la gran mayoría de mujeres exterminadas en esa época, se encontraban en situación de mayor vulnerabilidad social (solteras o viudas, sin hijos, de edades 
mayores, sin recursos económicos, sin nadie que ofreciese una protección sobre ellas) respecto al resto de la población.

Aunque cualquier persona pudiera ser acusada de brujería, era sobre las mujeres solas en quienes recaían con mayor facilidad los castigos, tanto por parte del pueblo como de los tribunales de la inquisición, el caso más evidente es el manual que con todo detalle, escribieron Kraemer y Sprenger llamado "El martillo de las brujas" en el que se especifica como lograr la confesión y exterminio de las mujeres practicantes de brujería ${ }^{5}$.

En el recorrido histórico que Fernández (2002: 304) realizó en torno a la historia de las mujeres españolas en el renacimiento, la figura de la bruja es rescatada de lo que aportan principalmente los novelistas de la época (p.e. La Celestina de Fernando de Rojas, y Coloquio de los Perros de Cervantes, entre otros), en donde se le atribuye a las mujeres-brujas un papel más de encubridora y curandera que de hechicera.

Este autor hace hincapié en las características físicas que les eran comunes a las brujas medievales y renacentistas “[...] viejas, feas, desastradas [...]” en contraste con una época que se recreaba en representar pictóricamente el desnudo femenino en toda su juventud, belleza y armonía.

De tal manera, que ni siquiera en el floreciente Renacimiento (ni ahora) se dejó de asociar vejez femenina con bruja repulsiva, como juventud femenina con belleza bondadosa. La imagen de "la bruja" es la de una mujer descuidada de su persona "achacosa", con carencias de todo tipo que la hacían repelente.

El mundo occidental siguió su curso, una vez apocados los saberes femeninos, la medicina galénica e hipocrática, manejada por el pensamiento "racional masculino" ocupó el escenario.

Hasta el siglo XVIII persistía la idea de que el alma debe controlar acéticamente al cuerpo, para evitar desajustes en la salud, por tanto se seguía considerando que las mujeres estaban relacionadas con la maternidad no por su

\footnotetext{
${ }^{5}$ Citados por Riquer, 1989:350-356.
} 
fisiología, sino por una "vocación metafísica del alma", lo que las liga ineludiblemente a la Naturaleza (Ariés y Duby, 1990).

Pero no fue hasta el siglo XIX, cuando surgieron las técnicas diagnósticas, o medicina anatomo-patológica, fundamentada en la idea de las alteraciones morfológicas, unificando medicina y fisiología, olvidándose deliberadamente de la preeminencia del alma. Todo aquello que escapaba a la explicación fisiológica pasó a ser parte del mundo de la psique, y por tanto sujeto a análisis también (Op. cit.).

La vida urbana de la pequeña burguesía se puso de moda, la higiene del cuerpo, la separación de lo animal y lo rústico por la pulcritud de la apariencia; las habitaciones privadas, el ejercicio al aire libre, la ducha, y el excusado. Se intentó desterrar todo aquello que significara un riesgo de contagio de enfermedades, a la vez que surgía la posibilidad de recuperar el culto al cuerpo como territorio íntimo e individualizado.

Todas estas prácticas que se instauraron desde la ciudad hasta el campo, propiciaron el logro de una vida más higiénica, lo limpio y ordenado se identificó como sinónimo de una buena moral. Sin embargo persistían creencias en torno a la moderación del baño, en detrimento de la higiene femenina, cuyo calendario menstrual seguía rigiendo la frecuencia de las duchas y de las relaciones sexuales.

Por su parte, los moralistas de la época mostraron un gran interés por controlar el "autoplacer" propiciado por los espacios que permitían privacidad en las personas (baños y recámaras principalmente) lo que desaceleró el avance de la profilaxis en materia de higiene sexual y propició una nueva disciplina sobre el cuerpo como territorio de placer sexual, obstaculizando (más no evitando) métodos para regular la fecundidad. (Ariés y Duby, $1990^{6}$ ).

A principios del siglo XX persistía la idea -aún entre los médicos- de que a las mujeres, la falta de embarazos sucesivos o de irrigación seminal en la vagina, les provocaba desequilibrio emocional, hemorragias y otras patologías (Op cit.). Lo que no evitó que muchas siguieran buscando opciones para regular su fecundidad -incluido el

\footnotetext{
${ }^{6}$ Véase también Foucault (1991), quien hace alusión a cómo la sociedad adquiere una doble moral ante las nuevas prácticas sociales emergentes.
} 
aborto- propiciando una solidaridad clandestina entre mujeres, pero también un incremento de padecimientos o incluso la muerte por prácticas riesgosas de interrupción de los embarazos.

Las prácticas y conocimientos que aún manejaban las mujeres, terminaron por ser acaparadas del todo por los médicos varones, a ellos se confiaba todo lo que pasa en la familia en materia de salud, atendiéndose expeditamente a sus consejos en cuanto a la maternidad, la puericultura, la pubertad, entre otros. ${ }^{7}$

El auge de las teorías anticontagionistas (gracias a los hallazgos de Koch y Pasteur principalmente) trajo consigo la consolidación de la medicina curativa, la pulcritud del médico y la sistematización de las terapias, donde el agua, el jabón y los antisépticos desplazaron a los viejos remedios, cobrando relevancia el diagnóstico y la catalogación de las enfermedades.

Esta etapa corresponde a la medicina de laboratorio del siglo $\mathrm{XX}$, en donde se comenzó a utilizar las pruebas o análisis para determinar el tipo de padecimiento presentado, instrumentos principales de la definición de salud y enfermedad hasta hoy en día, con base en clasificaciones estrictamente delimitadas (Bernabeu, Barona, Fernández y Robles, 1999); la etiqueta de normal y anormal, surge y prevalece hasta nuestros días.

Con todo lo antes descrito, no resulta difícil comprender cómo se ha "obligado" a las mujeres milenariamente a sanar y cuidar en el mundo privado, con lo que han aprendido por tradición oral de otras mujeres, pero considerando sus conocimientos "con poco valor, intuitivos y naturales".

Sayavedra y Flores (1997) mencionan que como resultado de esos episodios catastróficos contra las mujeres sanadoras, los médicos en Europa se apropiaron del saber curativo, restringiendo a las mujeres a la partería, oficio considerado de $2^{\mathrm{a}}$ categoría o importancia menor, ya que al incrementarse la participación de los cirujanos

\footnotetext{
${ }^{7}$ Véase al respecto Mazarrasa (2000).
} 
en los partos en el siglo XVIII, se fueron sentando los precedentes de la ginecología y la obstetricia, ejercidas principalmente por médicos varones.

En ese mismo sentido, Riquer (1989) concluye su trabajo con diversas reflexiones en torno a la necesidad de deconstruir las identidades de las mujeres, su relación con el cuerpo la sexualidad y la salud, a partir de la postura de la bruja, de la mujer que posee conocimientos, que se revela y que desafía al poder masculino dominante, pero ¿esto puede ser igual para las mujeres de todas las latitudes o de nueva cuenta las mujeres con menos poderes se quedarán a la saga?

\section{Las repercusiones en el caso de México}

Para el caso de México, incluso el trabajo de las parteras se vio restringido por la Práctica Médica Occidental. Sayavedra y Flores (1997) mencionan cómo se fue perdiendo la gran transmisión de los conocimientos de partería, confinando muchas veces esta práctica a ser ejercida clandestinamente, reconociendo solamente el trabajo de unas pocas mujeres.

Lo anterior no ha cambiado mucho en la actualidad, al considerarse en México (y gran parte de Latinoamérica) la partería $^{8}$ hoy en día como una práctica popular empírica y mucho más económica que una atención obstétrica "profesional"; de hecho, en regiones extremadamente pobres como el estado de Chiapas (equivalente a provincia), la mayoría de los partos son atendidos por parteras, ya que un parto atendido en una clínica particular puede costar $\$ 12800$ pesos mexicanos (753 euros), mientras que con una partera el costo puede ser de\$ 1280 pesos mexicanos (75 euros) o menos. Además la mayoría de las veces los servicios de salud pública están colapsados por

\footnotetext{
${ }^{8}$ En México actualmente solo existe una pequeña escuela de Partería en el país, y la profesión se oferta como una carrera a nivel técnico, con una colegiatura elevada para las posibilidades de la mayoría de la población. Las egresadas son aproximadamente 15 mujeres cada dos años, en un país con 4.200.000 embarazos al año.
} 
otras urgencias, por lo que las propias mujeres prefieren buscar alternativas, antes de ser rechazadas por los servicios de sanidad en pleno trabajo de parto.

Es así como, más allá de la Práctica Médica hegemónica, en los espacios de la subalternidad, la mayoría de las personas que procuran la salud son mujeres, por lo menos en el caso del México contemporáneo (parteras, promotoras de salud popular, curanderas, entre otras).

Dichas mujeres prestan sus servicios a colonias o comunidades por algún exiguo pago en dinero o en especie, o a cambio de una beca mínima, o incluso de manera gratuita. En un estudio realizado en el 2001 sobre el Programa de Ampliación de Cobertura Médica en Chiapas, se encontró que las becas pagadas por el ministerio de salud a las mujeres auxiliares de salud en las comunidades, no superaba los 20 euros mensuales, mientras que a las parteras no se les pagaba nada.

El trabajo realizado por estas mujeres puede resultar una contradicción ya que “[...] por un lado refuerza los roles asignados a las mujeres, aumenta su trabajo invisible como extensión de su trabajo de madresposas, pero por otro, las ha situado en la práctica política, en el ámbito de lo público [...]” (Sayavedra y Flores, 1997:79) dotándolas de cierto poder, pero sin un reconocimiento amplio de su trabajo, ya que se refuerza la idea de que las mujeres son las mayores responsables de la salud de su entorno (familiar y comunitario).

Lo anterior no es pasado por alto por la Secretaría de Salud (equivalente al ministerio de Sanidad en España), ya que se reconoce que muchos de los servicios de salud institucionales son proporcionados por mujeres (enfermeras, médicas, trabajadoras sociales, limpiadoras y otras profesionales paramédicas), pero que el número de mujeres en puestos de toma de decisiones es sumamente escaso, (SSA:2001) ejemplo de ello es que la Secretaría de Salud, nunca ha tenido como titular (o ministra) a una mujer. Así, a pesar de la actual definición de la OMS sobre el concepto de salud, la procuración de la salud de las mujeres en muchas regiones continúa en entredicho, como resultado de los valores que se reproducen y perpetúan. 
La medicina preventiva derivada de la Práctica Médica hegemónica, juega un papel esencial unido a las normas religiosas que han modelado los "valores aceptados", diluyendo la conciencia colectiva, regulando la vida privada de las personas y en particular, de las mujeres.

Coincidiendo con Sayavedra y Flores (1997) actualmente los procesos fisiológicos tales como la menstruación, el embarazo, el parto y la menopausia se siguen considerando sinónimos de enfermedad asumiéndose como "riesgos" para la salud de las mujeres; y esa visión a su vez, influye enormemente en lo que se concibe como salud por lo menos en el caso de las mujeres mexicanas, en donde se siguen presentando casos en los que a las mujeres se les extirpan los ovarios o el útero para prevenir padecimientos como el cáncer; o en donde mujeres de regiones pobres indígenas o mestizas son esterilizadas quirúrgicamente sin su consentimiento bajo el argumento de que ya han tenido demasiados hijos e hijas.

Así, los cambios a través del ciclo vital se viven con temores y culpas, sin una conciencia de apropiación corporal; otorgando con ello, a la medicina hegemónica, un gran control sobre el cuerpo femenino, afianzándose la contradicción de que las mujeres son las responsables de su salud pero a la vez su cuerpo no está bajo su propio control.

Lo anterior se puede constatar en diversos estudios realizados con mujeres que han pasado la etapa reproductiva -mayores de 40 años- quienes tienen serios problemas para responsabilizarse de su autocuidado y la prevención de su salud, a la par de que dentro del sistema sanitario mexicano, la figura de las mujeres en etapas posteriores al ciclo de reproducción biológica, está invisibilizada (Sánchez, 2004a; Sánchez, 2004b y Sánchez, en prensa).

En numerosas ocasiones han influido también las políticas de población que "prescriben" medidas que afectan directamente al cuerpo y las capacidades biológicas de las mujeres, (disminuir la fecundidad, aplazar los embarazos, evitar la mortalidad materna por el "costo" económico y social que representa, etc.) y donde el poder médico se encargará de "vigilar" que dichas disposiciones se lleven a cabo, sin que ello pase por un proceso de conciencia para las mujeres de los países del Tercer Mundo. 


\section{La necesidad de nuevas relaciones entre prestadores de servicios de salud y usuarias}

Por parte de los profesionales en medicina, comienzan a escucharse posturas críticas respecto al derecho y autonomía de los y las pacientes, principalmente en Europa y Estados Unidos de Norteamérica, de tal manera que el cuerpo (de mujeres y varones) no sea visto como un receptáculo pasivo de la práctica médica.

López de la Vieja (2001) refiere que en occidente se ha transitado de una medicina hipocrática extremadamente paternalista, (donde la autonomía solo se reconocía en el profesional de medicina) a una medicina en la que es cada vez más importante el reconocimiento del paciente como sujeto, cuya opinión es determinante en el camino a seguir para su tratamiento o recuperación.

En ese mismo sentido Abel (2002) menciona que para aminorar la desconfianza o distancia que se ha creado entre profesionales médicos y pacientes es necesario enfatizar el respeto al paciente como persona, la delicadeza en el trato y el esfuerzo por parte del servidor para mantener la competencia profesional a través de la formación continua.

Esta autora menciona también que debido a que la relación médico-paciente ha pasado del ámbito privado al de la salud pública, es necesario considerar factores como la financiación del sector (recortes de presupuesto, prioridades de atención, etc.) y la creciente tendencia de "mercantilizar" la salud, como una conjunción de elementos que deben ser regulados de una manera ética, para el logro de una buena atención en salud ${ }^{9}$. De esta manera existen dos factores que se entraman para marcar las pautas de una atención en salud adecuada, el compromiso de los profesionales de la medicina donde principalmente se reconozca la autonomía de los y las pacientes y el reconocimiento de las mujeres por sí mismas como personas sujetas de derechos.

\footnotetext{
${ }^{9}$ Véase también Leal y Martínez (2002), quienes documentan las reformas que el sector salud mexicano ha tenido de cara a abaratar costos en detrimento de la calidad de la atención.
} 
En el caso de México, las relaciones médico-paciente siguen estando apegadas al modelo paternalista, con muy poco reconocimiento de la autonomía, principalmente en las pacientes mujeres; unido al escaso compromiso por la formación continua, que favorece, por ejemplo que, muchos médicos varones, prescriban fármacos a las mujeres climatéricas, sin tomar en cuenta la necesidad de personalizar los tratamientos de acuerdo a la historia de vida de cada mujer (Sánchez, 2006).

Esto está relacionado también con el perfil de la población atendida. La salud de la mayoría de las mujeres en el país sigue dependiendo de una u otra manera del cuidado de los médicos: ya sea por una incuestionable confianza que deja que ellos decidan sobre su cuerpo -cesáreas, esterilizaciones quirúrgicas, histerectomías-, o bien evitando una atención médica oportuna, debido a que desconfían totalmente de los servicios o de la figura médica, tanto en la sanidad pública como en la privada.

Tampoco ayuda el hecho de que en el país sean cada vez más mujeres las que egresen de las carreras de medicina, ya que no necesariamente están incorporando el concepto de género y salud; las currículas de las asignaturas orientadas hacia una biomedicina occidental hegemónica; sin cuestionamientos respecto al androcentrismo científico; y sin incorporar elementos de otro tipo de conocimientos en salud como en el caso de China por ejemplo, a pesar de que como ya se ha mencionado en México la presencia de la herbolaria y otras alternativas de curación están presentes en toda la latitud mexicana, lo cual también implicaría un rescate de los saberes alternativos sobre salud; de una manera sistematizada, pero sin enajenar a los y las conocedoras de conocimientos; sino como una verdadera deconstrucción de la medicina en el país, lo cual también implicaría un poco de humildad de parte de la medicina institucional; al reconocer en la otra el conocimiento.

En la medida en que las mujeres (pacientes y profesionistas o prestadoras de servicios de salud) tengan elementos de asertividad - los cuales dependerán de su escolaridad, clase social, etnia, religión, edad, nacionalidad, información y redes de apoyo a disposición-, la relación que se establezca entre ellas y el servicio médico tendrá mayor o menor asimetría de poder, propiciando un lazo de verdadera atención y 
comunicación que logre la resolución o prevención del problema de salud por el que acudan a los servicios médicos.

\section{Aportaciones del enfoque de Género y Salud}

Ante este panorama y para finalizar no se quiere dejar de mencionar que han surgido otras alternativas contemporáneas para procurar la salud de las mujeres, donde ellas mismas sean sujetos activos de un verdadero autocuidado, desde la dimensión de salud tal y como lo propone la Organización Mundial de la Salud, de la forma en la que se citó en la introducción de este artículo.

En ello han jugado un papel determinante, las reivindicaciones que los movimientos feministas desde la década de los 50 han venido haciendo respecto a los derechos de las mujeres y la reivindicación de su autonomía, derivándose en enfoque loables como el enfoque de Género y Salud, cuya mayor aportación al analizar la salud, es la posibilidad de distinguir la diferencia en el enfermar y el sanar de mujeres y varones. Ello recae tanto en las diferencias biológicas, fisiológicas y morfológicas entre los sexos como en las situaciones, condiciones o problemas de salud disímiles para cada género.

Este enfoque reconoce además las diferentes necesidades de salud entre varones y mujeres, y las desiguales condiciones en las que las mujeres enfrentan y resuelven sus problemas de salud a lo largo de todo su ciclo vital, principalmente en los contextos de los países en vías de desarrollo; por tanto un factor muy importante es favorecer condiciones que permitan a las mujeres decidir por sí mismas sobre su salud.

Lo anterior es una meta en extremo ambiciosa y que debe de contemplar en primera instancia, la realidad de las mujeres a las que se dirijan las intervenciones en salud. Para el caso de las mujeres de regiones en vías de desarrollo como México, en donde sus condiciones de salud suelen ser el reflejo de la escasez de recursos, falta de cobertura y acceso de los servicios, aunado a toda una serie de obstáculos para tomar 
sus propias decisiones debido a la subordinación frente a los varones, es imprescindible tomar estos elementos en cuenta para el diseño de acciones de desarrollo y salud.

Se tiene entonces que la perspectiva de género y salud debe de considerar, como ya se ha mencionado al principio de este capítulo, elementos que influyen en el status quo:

"Son tan complejas las articulaciones entre desigualdad de género y otras formas de desigualdad social, y entre poder de género y otras relaciones de poder, que cualquier intento de considerar las interrelaciones entre género y salud requiere de la concreción del contexto espacial, temporal, histórico y sociocultural, y de experiencias subjetivas en las que ocurren esas relaciones [...]" (Szasz, 1999: 118).

Por tanto el abordaje de género se debe dirigir hacia la transformación social y no limitarse a incrementar recursos para las mujeres. Las estrategias de Políticas Públicas desde el enfoque de género y salud deben de ser una síntesis entre lo observado e interpretado a nivel individual y colectivo para plantear modificaciones a la realidad, a través de procesos continuos, con el fin de detectar y corregir las asimetrías entre los géneros, las relaciones de poder e inequidad y sus efectos adversos o protectores en las personas (Velasco, 2002).

No obstante, en el caso de México no solo se está lejos de lograr los objetivos de salud para todas y todos, sino que además se está dando marcha atrás a compromisos contraídos como en la Conferencia de Población y Desarrollo del Cairo en 1994, al vulnerarse actualmente leyes y códigos que protegían la salud sexual de las mujeres; al persistir graves indicadores de mortandad materna; y elevados índices de cáncer de cérvix y de seno, entre otros indicadores que vulneran la condición de las mujeres (PNUD; 2009), convirtiéndose éstas en seres enajenados del conocimiento y control de sus propios cuerpos y de su salud. 


\section{Conclusiones}

La racionalidad técnica de la medicina hegemónica, si bien ha traído aportes sin precedentes a la salud la ha colocado como un producto consumible solamente por quienes tienen y pueden acceder a ella, negando además el reconocimiento de otro tipo de saberes o conocimientos en torno a la procuración de la salud, a lo que subyace además una lucha de poderes de reconocimiento entre géneros: lo que se considera como verdadero sigue procediendo de la visión de los médicos varones o del saber médico masculinizado. Todo aquello que ayuda en la preservación de la salud (cuidados) se atribuye de manera natural a las mujeres como resultado de la división sexual del trabajo y de los roles tradicionales de género, además los saberes de las mujeres que procuran la salud no se encuentran reconocidos en el rango de valoración de la medicina hegemónica, ni siquiera en contextos pobres como México en el que gracias a esos conocimientos y cuidados de las mujeres procuradoras de salud (ya sea para su familia o para toda su comunidad) la población puede tener cierto equilibrio entre salud y enfermedad.

En cuanto a la autonomía y el conocimiento del propio cuerpo de las mujeres, hemos perdido el conocimiento milenario de las brujas sobre nuestros ciclos vitales, nuestra sexualidad y nuestras emociones; no obstante tenemos un nuevo milenio para hallarlo, hacernos nuestras, dueñas de sí, enfrentando a los nuevos inquisidores con el valor y la audacia de las belladonas.

\section{Agradecimientos}

A Gabriela Mariana Fenner Sánchez por la revisión y sugerencias a este documento. 


\section{BIBLIOGRAFÍA}

- Abel, F. (2002): "Ética de la profesión médica". En Gómez-Heras J. (coord.): Dignidad de la vida y manipulación genética. Bioética. Ingeniería genética. Ética feminista. Deontología Médica. España: Biblioteca Nueva, pp. 237-281.

- Aguirre, H. et al. (1989): La medicina Tradicional en México y su supervivencia. México: Universidad Nacional Autónoma de México.

- Ariés, P. y Duby, G. (1990): Historia de la vida privada. De la revolución francesa a la $1^{a}$. Guerra Mundial. España: Ed. Taurus.

- Bernabeu, J.; Barona, J.; Fernández E. y Robles E. (1999): “La Historia Social a través de la enfermedad y sus manifestaciones: mortalidad y morbilidad". En Pérez Pilar (comp.): Didáctica de la demografía histórica. Actas del Congreso Internacional de la Población. V Congreso de la ADEH. Vol. V. España: pp. 99-125.

- Blazquez, N. (2008): El retorno de la bruja. México: UNAM-CIICH.

- Breilh, J. (1988): Epidemiología. Economía. Medicina y Política. México: Fontamara.

- Esteban, M. (2001): “El género como categoría analítica. Revisiones y aplicaciones a la salud". En Miqueo C. et al. (eds.): Perspectivas de Género en Salud. Fundamentos científicos y socioporfesionales de diferencias sexuales no previstas. España: Minerva ediciones, pp. 25-51.

- Fernández, M. (2002): Casadas, Monjas, Rameras y Brujas. La olvidada Historia de la mujer española en el Renacimiento. España: Espasa.

- Foucault, M. (1991): Historia de la sexualidad, vol I. México: Siglo XXI.

- Greer, G. (1993): El cambio. Mujeres, vejez y menopausia. España: Anagrama.

- Guzmán, M. (1987): Prácticas médicas en la América antigua, 2a imp. México: Ediciones Euroamericanas.

- Harris, M. (1981): “Escobas y Aquelarres”. En Vacas, cerdos, guerras y brujas. Los enigmas de la cultura. España: Alianza Editorial, pp. 181-193.

- Laurell, C. (1982): "El carácter social del proceso salud-enfermedad y su relación con el proceso de trabajo". En Ortiz F.: Vida y muerte del mexicano. México: Folios Editores. 
- Leal, G. y Martínez, C. (2002): Cuatro ensayos sobre calidad clínica de la atención. El caso de la salud reproductiva. Serie Académicos CBS. México: Universidad Autónoma Metropolitana.

- López de la Vieja, T. (2001): Principios morales y casos prácticos. España: Tecnos.

- Marcos, S. (1989): “Las curanderas mexicanas”. En O. Oliveira (coord.): Trabajo, poder y sexualidad. México: El Colegio de México, pp. 359-382.

- Marset, P. y Sáez, M. (2002): "Mujeres y salud a lo largo de la historia”. En E. Ramos (coord.): La salud de las mujeres: hacia la igualdad de género y salud (I Congreso Nacional sobre Salud y Mujeres). España: Instituto de la Mujer, p. 66-85.

- Matamala, M. (1995): Calidad de la Atención. Género: ¿Salud Reproductiva de las mujeres? Chile: CONAMUS-ACHNU.

- Mazarrasa, L. (2000): "Rol de género en la relación entre el personal de enfermería y el personal médico". En Género y Salud. I Jornadas de la Red de Médicas y Profesionales de la Salud. España: Ministerio de Asuntos Sociales/Instituto de la Mujer, pp. 33-36.

- Menéndez, E. (1983): "Recursos y prácticas médicas tradicionales". En Lozoya y Zolla: La medicina invisible. México: Folios Editores.

- Michelet, J. (1987): La bruja. Un estudio sobre las supersticiones en la Edad Media. España: Editorial Akal.

- Murillo, S. (1996): El mito de la vida privada. De la entrega al tiempo propio. España: Siglo XXI.

- OMS - Organización Mundial para la Salud (1987): Boletín de la Oficina Panamericana, $\mathrm{n}^{\circ} .103$.

- Ortega, P. y Rodríguez, M. (ed.) (1998): Mujer, Ideología y Población. España: Ed.. Clásicas S.A.

- Pardo, E. (1998): "Medicina oficial y modelo alternativo" En Natura Medicatrix no. 58, Octubre.

- PNUD - Programa de las Naciones Unidas para el Desarrollo (2009): "Informe sobre desarrollo humano". México, [en línea] Disponible en http://hdr.undp.org/en/media/HDR_2009_ES_Complete.pdf. [Acceso 15/02/2010]. 
- Riquer, F. (1989): "Brujas e identidad femenina". En O. Oliveira (coord.) Trabajo, poder y sexualidad. México: El Colegio de México, pp. 331-358.

- Rojas, R. (1985): Capitalismo y enfermedad. México: Folios Ediciones.

- Sanginés, A. (1990): Medicina Liberadora. Teoría método y práctica. México: Ed. Praxis.

- Sayavedra, G. y Flores, E. (1997): Ser mujer ¿un riesgo para la salud? Del malestar y el enfermar al poderío y la salud. México: Red de Mujeres, A.C.

- Sayavedra, G. (1982): Práctica médica educativa dominante y subalterna. Tesis de Maestría en Ciencias. México: Instituto Politécnico Nacional-DIE.

- Sánchez, G. (2006): Incidencia de la Información en Salud y Sexualidad desde el enfoque de género en la calidad de vida en el climaterio, en tres regiones de México (estado de México, Veracruz y Chiapas). Tesis Doctoral. España: Universidad de Salamanca, Facultad de Medicina.

- Sánchez G. (2004a): "Empowerment, health and aging in Mexican women". En Women's Health Journal, no. 4, pp.59-68.

- Sánchez G. (2004b): “Ni jóvenes ni ancianas. La atención en el Climaterio. El Caso de tres regiones de México”. En Revista GénEros, año. 11, n. 32, pp.33-41.

- Sánchez G. (en prensa): "Realidades y mitos sobre la salud y la sexualidad en la madurez femenina”. En Anuario del CESMECA 2009, México. CESMECA-UNICACH. - SSA - Secretaria de Salud (2001): "Inaplazable incorporar la perspectiva de género en las políticas públicas de salud”. En Comunicado de prensa, nº 185, 26 de Noviembre de 2001. México D.F.

- Sendón, V. (1988): Más allá de Itaca. Sobre complicidades y conjuros. España: Editorial Icaria.

- Szasz, I. (1999): “Género y salud. Propuestas para el análisis de una relación compleja". En M. Brofman y R. Castro (coord.): Salud, Cambio Social y Política. Perspectivas desde América Latina. México: Edamex, pp. 109-121.

- Velasco, S. (2002): Relaciones de género y subjetividad. Método para programas de prevención. Serie Salud, vol. 5. España: Instituto de la Mujer. 